\title{
A BRIEF STUDY ON ENERGY IN ITINERANT-ELECTRON METAMAGNETIC MATERIALS AT VERY LOW TEMPERATURE
}

\author{
M. A. GRADO-CAFFARO* and M. GRADO-CAFFARO \\ C./Julio Palacios, $11,9^{\circ} \mathrm{B}, 28029-$ Madrid (Spain)
}

(Received 4 July 1996; In final form 30 August 1996)

\begin{abstract}
Electronic energy is calculated explicitly for itinerant-electron metamagnetic materials at very low temperature. This calculation involves bandwidth and consequently volume, and it has been performed by means of an elliptic density of states. Moreover, total energy is considered.
\end{abstract}

\section{INTRODUCTION}

Problems associated with itinerant-electron-metamagnetism offer a wide research field with a number of unsolved questions; in fact, both theoretical and experimental work in the above field seems to be scant. One of the subjects involved in the mentioned phenomenon is the electronic energy; this energy plus the lattice energy gives the total energy. Electronic energy depends on the volume through the bandwidth [1][2][3][4], and the lattice energy depends also on volume [1][3]. In the following, we shall derive an expression for the electronic energy of itinerant-electron metamagnetic materials at $T \approx 0^{\circ} \mathrm{K}$ in the context of the Stoner approach; this relationship will be expressed in terms of volume, and it is particularly useful to study vanadium oxide in which a transition between the metallic and the

*Corresponding author. 
antiferromagnetic insulating phases occurs with volume discontinuity [1][5]. With respect to this, we recall that magnetic field, pressure, and temperature may be varied to originate a first-order transition between a non-magnetic state and a ferromagnetic one [2][6]; this transition characterizes itinerant-electron metamagnetism. This phenomenon has been observed in several rare-earth intermetallic compounds [7][8].

\section{THEORY}

Now, in accordance with the Stoner model, the electronic energy at $T \approx$ $0^{\circ} \mathrm{K}$ reads [1][3]:

$$
E_{e}=\int_{-W}^{E_{F 1}} E g(E) d E+\int_{-W}^{E_{F 2}} E g(E) d E-\frac{1}{4} J M^{2}-\mu_{B} M H
$$

where $E$ denotes energy, $2 W$ is the involved bandwidth, $g(E)$ is the density of states, $E_{F 1}$ and $E_{F 2}$ are the Fermi levels for the up and down spin bands respectively, $J$ is the exchange energy between up and down spin electrons, $\mu_{B}$ is the Bohr magneton, $M$ denotes magnetization, and $H$ is the strength of an applied magnetic field. With an elliptic density of states $g(E) \approx 3\left(W^{2}-E^{2}\right)^{1 / 2} / W^{2}[1][2][3]$, expression (1) becomes:

$$
\mathrm{E}_{\mathrm{e}}(\mathrm{W}) \approx-\frac{\left(\mathrm{W}^{2}-\mathrm{E}_{\mathrm{F} 1}^{2}\right)^{3 / 2}+\left(\mathrm{W}^{2}-\mathrm{E}_{\mathrm{F} 2}^{2}\right)^{3 / 2}}{\mathrm{~W}^{2}}-\frac{1}{4} \mathrm{JM}^{2}-\mu_{\mathrm{B}} \mathrm{MH}
$$

Note that (since $J>0$ ) $E_{e}<0$ by simple inspection of eq. (2). On the other hand, if $E_{F 1} \ll W$ and $E_{F 2} \ll W$, from eq. (2) it is deduced that $\mathrm{E}_{e}(\mathrm{~W}) \approx-2 \mathrm{~W}-\frac{1}{4} \mathrm{JM}^{2}-\mu_{\mathrm{B}} \mathrm{MH}$; this situation is interesting in certain cases. Now, let us consider the well-known Slater-Koster formula, namely [4]:

$$
\mathrm{W}=\mathrm{W}_{\mathrm{o}} \exp \left[-\alpha\left(\mathrm{V}-\mathrm{V}_{\mathrm{o}}\right) / \mathrm{V}_{\mathrm{o}}\right]
$$

where $V$ denotes volume, $W_{o}$ is the value of $W$ for $V=V_{o}$, and $\alpha$ is a parameter such that $1 \leq \alpha \leq 5 / 3$; formula (3) was obtained by using a tight-binding approach for $3 \mathrm{~d}$-electrons. For very small volume change, 
that is, for $V-V_{o}<V_{o}$, eq. (3) becomes (by considering a first-order McLaurin expansion):

$$
\mathrm{W} \approx \mathrm{W}_{\mathrm{o}}\left[1-\alpha\left(\frac{\mathrm{V}-\mathrm{V}_{\mathrm{o}}}{\mathrm{V}_{\mathrm{o}}}\right)\right]
$$

Putting $\alpha=3 / 2$ into (4), it follows:

$$
\mathrm{W} \approx \frac{1}{2} \mathrm{~W}_{\mathrm{o}}\left(5-\frac{3 \mathrm{~V}}{\mathrm{~V}_{\mathrm{o}}}\right)
$$

Expression (5) is the Grado-Grado formula [2][3] which has been obtained by means of a quantum-mechanical treatment involving a tightbinding hamiltonian and a perturbation method. By replacing (5) into (2), we get:

$$
\mathrm{E}_{\mathrm{e}}(\mathrm{V}) \approx-\frac{4}{\mathrm{~W}_{\mathrm{o}}^{2}}\left\{\frac{\left[\frac{\mathrm{W}_{\mathrm{o}}^{2}}{4}\left(5-\frac{3 \mathrm{~V}}{\mathrm{~V}_{\mathrm{o}}}\right)^{2}-\mathrm{E}_{\mathrm{F} 1}^{2}\right]^{3 / 2}+\left[\frac{\mathrm{W}_{\mathrm{o}}^{2}}{4}\left(5-\frac{3 \mathrm{~V}}{\mathrm{~V}_{\mathrm{o}}}\right)^{2}-\mathrm{E}_{\mathrm{F} 2}^{2}\right]^{3 / 2}}{\left(5-\frac{3 \mathrm{~V}}{\mathrm{~V}_{\mathrm{o}}}\right)^{2}}\right\}
$$

We have deduced $\mathrm{E}_{\mathrm{e}}(\mathrm{W}) \approx-2 \mathrm{~W}-\frac{1}{4} \mathrm{JM}^{2}-\mu_{\mathrm{B}} \mathrm{MH}$ when $E_{F 1}<W$ and $E_{F 2}<\ll$. Inserting (5) into the preceding formula, it follows:

$$
\mathrm{E}_{\mathrm{e}}(\mathrm{V}) \approx-\mathrm{W}_{\mathrm{o}}\left(5-\frac{3 \mathrm{~V}}{\mathrm{~V}_{\mathrm{o}}}\right)-\frac{1}{4} \mathrm{~J} \mathrm{M}^{2}-\mu_{\mathrm{B}} \mathrm{MH}
$$

In order to preserve the negative sign of the first term in the right-hand side of eq. (7) ( $E_{e}$ must be negative), the following condition is obtained:

$$
\frac{\mathrm{V}}{\mathrm{V}_{\mathrm{o}}} \leqslant \frac{5}{3}
$$


Thus, it is feasible to formulate the Grado-Grado theorem of itinerantelectron metamagnetism as follows: at $T \approx 0^{\circ} \mathrm{K}$, for itinerant-electron metamagnetic materials with $E_{F 1}, E_{F 2} \ll W$ and very small volume change, the approximate inequality (8) is satisfied.

Finally, we shall refer to lattice energy $E_{\ell}$. We have $E_{t}=E_{e}+E_{\ell}$ where $E_{t}$ stands for total energy; since $E_{\ell}$ depends on volume variation, we can claim that $E_{\ell} \ll E_{e}$ for transitions with very small volume change [1][3] so that one has $E_{t} \approx E_{e}$.

\section{CONCLUSION}

In conclusion, we can claim that although analytic treatments based on an elliptic density of states lead to results that are not exactly valid for intermetallic compounds, these treatments agree qualitatively with experiment [1][3][9]. We have performed various calculations in order to establish negativeness of the electronic energy studying the situation in which volume variation is very small as a relevant case. Furthermore, $E_{F 1}<W$ and $E_{F 2}<\ll W$ have been considered so that inequality (8) has been obtained. Expression (8) is valid for, say, a typical itinerant-electron metamagnetic material.

\section{References}

[1] Duc, N. H., Givord, D., Lacroix, C. and Pinettes, C. (1992). Europhys. Lett., 20, $47-52$.

[2] Grado-Caffaro, M. A. and Grado-Caffaro, M. (unpublished),

[3] Grado-Caffaro, M. A. and Grado-Caffaro, M. (1994). Act. Pass. Electronic Comp., 17, $185-187$.

[4] Slater, J. C. and Koster, G. (1954). Phys. Rev., 94, 1498.

[5] Cyrot, M. and Lacour-Gayet, P. (1972). Solid State Commun., 11, 1767.

[6] Shimizu, M. (1981). Rep. Prog. Phys., 44, 329.

[7] Lemaire, R. (1966). Cobalt, 33, 201.

[8] Petrich, G. and Mössbauer, R. L. (1968). Phys. Lett., A 26, 403.

[9] Yoshimura, K., Shiga, M. and Nakamura, N. (1986). J. Phys. Soc. Jpn., 55, 3585. 

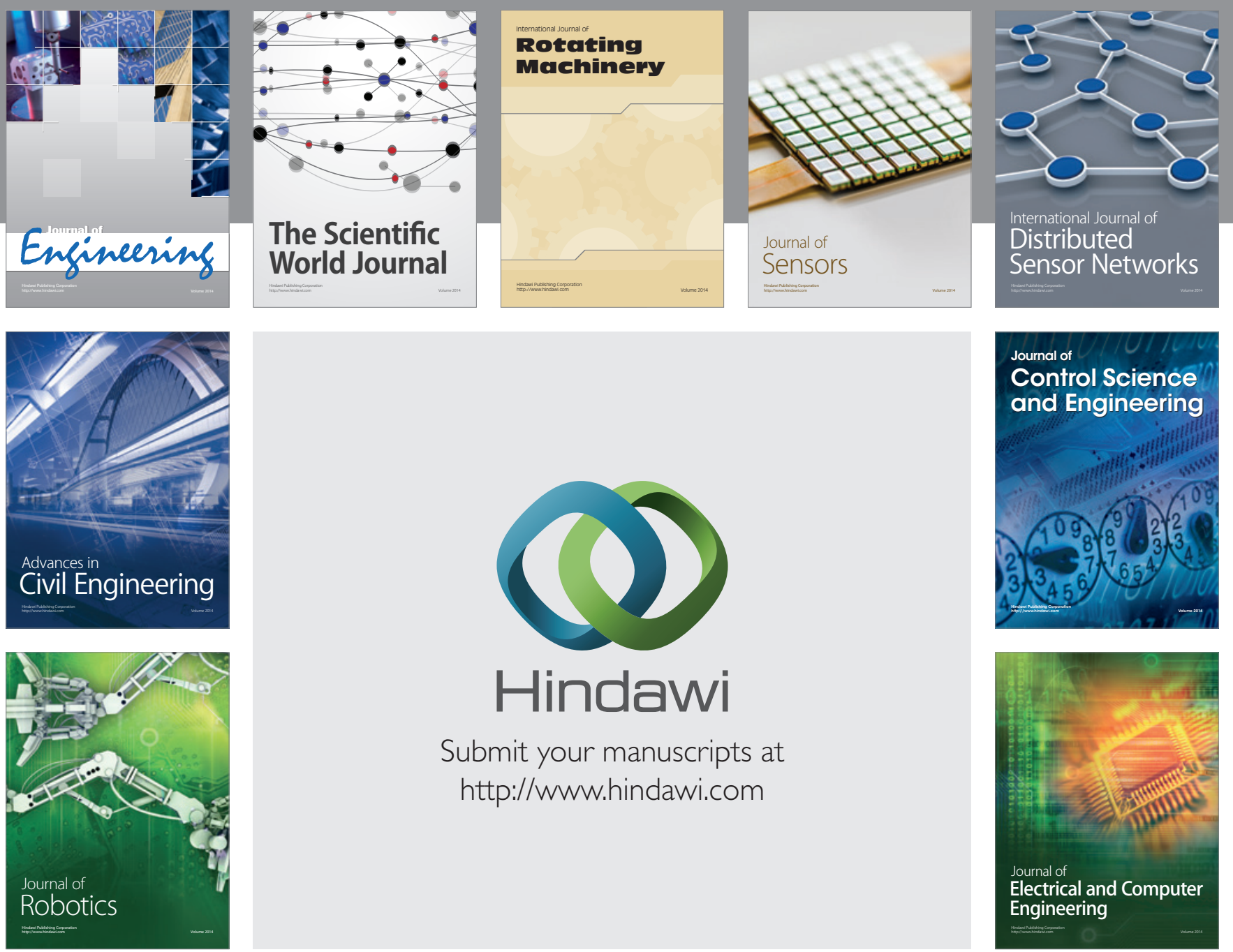

Submit your manuscripts at

http://www.hindawi.com
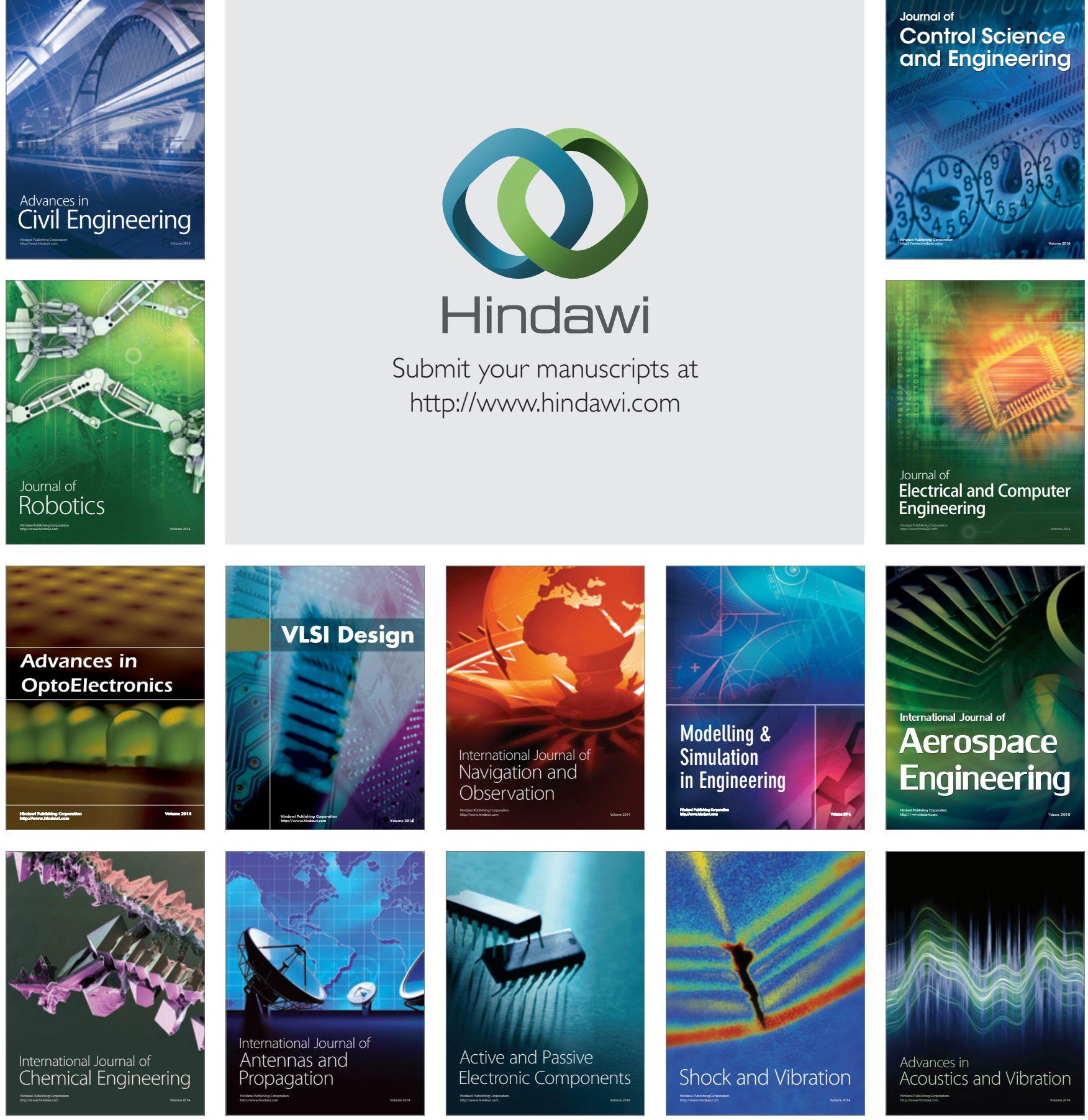